\title{
An Improved ICI Self Cancellation Scheme for OFDM Systems Under Various Channels
}

\author{
A.H. Sharief*, M. Satya Sai Ram** \\ *Department of ECE, Gandhiji Institute of Science and Technology, Andhra Pradesh, India \\ ** Department of ECE, Chalapathi Institute of Engineering and Technology, Guntur, India
}

\section{Article Info \\ Article history: \\ Revised Dec 7, 2015 \\ Keyword: \\ Frequency Offset \\ ICI \\ OFDM \\ Rayleigh channel \\ Rician channel}

Received Sep 23, 2015

Accepted Dec 28, 2015

\section{Corresponding Author:}

A.H. Sharief,

Department of Electronics and Communication Engineering,

Gandhiji Institute of Science and Technology,

Jaggayyapeta, Andhra Pradesh, India.

Email: shariefah@gmail.com

\begin{abstract}
Inter Carrier Interference (ICI) is being introduced in OFDM due to the carrier frequency offset (CFO), which will degrade the system performance and efficiency at higher modulation levels and it decreases the performance of power amplifiers. Hence, here in this paper, we introduced a novel ICI reduction algorithms cancellation under the various channel environments such as AWGN, Rayleigh and also Rician. Simulation results have been compared with existing and proposed schemes under these channel specifications and concluded that the Rayleigh has performed far better than the AWGN and Rician channel distributions in terms of Bit Error Rate (BER) and Carrier interference Ration (CIR) performance.
\end{abstract}

Copyright () 2016 Institute of Advanced Engineering and Science. All rights reserved.

\section{INTRODUCTION}

Orthogonal Frequency Division Multiplexing (OFDM) is being used for high data rate wireless applications [1]. It is amulticarrier modulation technique which incorporatesorthogonal subcarriers. High Peak to Average Power ratio andInter Carrier Interference (ICI) is two main disadvantages ofthe OFDM systems. In OFDM systems ICI occurs due tofrequency offset in between the transmitter and receivercarrier frequencies or Doppler Effect [2]. Many techniques have been developed to reduce the effect of ICI; ICI self cancellation is a simple and convenient technique. ICI self cancellation scheme proposed by Zhao [3] utilizesdata allocation and combining of $(1,-1)$ on two adjacent subcarriers i.e. same data is modulated at $k^{\text {th }}$ and $k+1^{\text {th }}$ sub carriers using $(1,-1)$ as data allocation and are combined at the receiver with weights 1 and -1. It is one of the mostpromising techniques to reduce ICI; however, its performancedegrades at higher frequency offsets. Yeh, Chang and Hassibihad proposed conjugate cancellation scheme in [4]. In this scheme, OFDM symbol and its conjugate are multiplexed, transmitted and combined at the receiver to reduce the effect of ICI. However, this scheme shows a significant improvement in CIR at very low frequency offsets and its performance degrades as carrier frequency offset increases. At higher frequency offset $>0.25$ its CIR performance is worse than standard OFDM system. In [5] the author has proposed Phase Rotated Conjugate Cancellation (PRCC), which is an extension to the scheme proposed in [4]. In this an optimal value of phase is multiplied with the OFDM symbol and its conjugate signal to be transmitted on different path. Theoptimal value of the phase depends on the frequency offset and hence requires continuous carrier frequency offset (CFO) estimation and feedback circuitry, which increases thehardware complexity [6-7]. Another ICI self cancellation scheme [8] based ongeneralized data allocation $\left(1, \mu e^{j \theta}\right)$ has been proposed in the literature to improve CIR performance of ICI self cancellationsystem, where $\xi$ is the optimal value, which depends on 
frequency offset. Thus for every normalized frequency offset, a unique value of $\xi$ is to be multiplied with the data which again requires CFO estimation and feedback circuitry [6-7]. A symmetric symbol repeat ICI self cancellation scheme, which utilizes data allocation and combining of $(1,-1)$ at $k^{\text {th }}$ and $\mathrm{N}-1-k^{\text {th }}$ subcarrier. This scheme shows better CIR performance than ICI self cancellation scheme. One of the major advantages of this scheme is to achieve the frequency diversity and hence its performance in frequency selective fading channel found to be better than ICI self cancellation scheme. In this paper, we have proposed an optimaland sub-optimal scheme for SSR ICI cancellation scheme to improve the CIR performance. The scheme is based on SSR ICI self cancellation scheme, in which a data is modulated at two symmetrically placed subcarriers i.e. $k^{\text {th }}$ and N-1- $k^{\text {th }}$ and utilizes a data allocation of $(1,-\lambda)$ to improve CIR performance. To further reduce the effect of ICI, received modulated data signal at $k^{\text {th }}$ and $\mathrm{N}-1-k^{\text {th }}$ subcarriers are combined with weights 1 and $-\xi$. The $\lambda$ and $\xi$ are the optimal values resulting in maximum CIR. The optimum values of $\lambda$ and $\xi$ are the function of normalized frequency offset i.e. for every normalized frequency offset; there exist a unique value of $\lambda$ and $\xi$. This process requires continuous CFO estimation. To overcome this problem, we have proposed a suboptimal approach to find suboptimal values. The obtained sub-optimal values $\left(\lambda_{s o}, \xi_{s o}\right)$ are independent of normalized frequency offset. Thus, the proposed scheme does not require any CFO estimation or feedback circuitry and hence eliminates the requirement of complex hardware circuitry.

\section{EXISTING METHODS}

\subsection{OFDM System}

The discrete time OFDM symbol at the transmitter can be expressed as

$$
x[n]=\frac{1}{\sqrt{N}} \sum_{k=0}^{N-1} X(k) e^{\frac{j 2 \pi n k}{N}}, n=0,1,2, \ldots \ldots, N-1
$$

where $\mathrm{N}$ is total numbers of subcarriers and $\mathrm{X}(\mathrm{k})$ denotes the modulated data symbol transmitted on $k^{\text {th }}$ subcarrier. Due to AWGN channel and frequency offset, the received OFDM signal can be written as

$$
y[n]=x[n] e^{j-\frac{2 \pi \varepsilon n}{N}}+w[n], n=0,1,2, \ldots \ldots \ldots, N-1
$$

where $\varepsilon$ is the normalized frequency offset and $w[n]$ is the sample of additive white Gaussian noise. The received data signal on $k^{\text {th }}$ subcarrier can be written as

$$
Y(k)=X(k) S(0)+\sum_{l=0, l \neq k}^{N-1} X(l) S(l-k)+W(k), k=0,1, \ldots ., N-1
$$

Where $W(k)$ is $k^{t h}$ the sample of DFT of additive noise. The sequence $S(l-k)$ is defined as the ICI coefficient between $k^{t h}$ and $l^{t h}$ subcarriers, which can be expressedas

$$
S(l-k)=e^{\left(j \pi(l+\varepsilon-k)\left(1-\frac{1}{N}\right)\right)} \frac{\sin (\pi(l+\varepsilon-k))}{N \sin \left(\frac{\pi}{N}(l+\varepsilon-k)\right)}
$$

The CIR at the $k^{\text {th }}$ subcarrier can be written as

$$
C I R=\frac{|S(k)|^{2}}{\sum_{l=0, l \neq k}^{N-1}|S(l-k)|^{2}}
$$

\section{A. SSR ICI Self Cancellation Scheme}

In SSR ICI self cancellation scheme [6], the data symbol to be transmitted at the $k^{\text {th }}$ subcarrier is repeated at the subcarrier $N-1-k^{\text {th }}$ with opposite polarity, i.e.,

$$
X(N-1)=-X(0), \ldots, X(N-1-k)=-X(k)
$$

The block diagram of the proposed SSR ICI self cancellation scheme is depicted in Figure 1. The received data signal at the $k^{\text {th }}$ subcarrier is thus given by

$$
Y^{\prime}(k)=\sum_{i=0}^{\frac{N}{2}-1} X(l) S((l-k)-S(N-1-l-k))+W(k)
$$


Combining the received data at $k^{\text {th }}$ and $N-1-k^{\text {th }}$ subcarriers, we have

$$
Y^{\prime \prime}(k)=Y^{\prime}(k)-Y^{\prime}(N-1-k)
$$

Using (6) \& (7) we have

$$
\begin{aligned}
& Y^{\prime \prime}(k)=\sum_{l=0}^{\frac{N}{2}-1} X(l)[S(l-k)-S(N-1-l-k)-S(l+k+1-N)+S(k-l)+W(k)-W(N \\
& k=0,1,2, \ldots ., \frac{N}{2}-1
\end{aligned}
$$

Thus, CIR of conventional SSR ICI self cancellation scheme can be written as

$$
C I R_{C}=\frac{|-S(-N-1-2 k)+2 S(0)-S(1-N+2 k)|^{2}}{\sum_{l=0, l \neq k}^{\frac{N}{2}-1}|-S(l-k)-S(N-1-l-k)-S(l+k+1-N)+S(k-l)|^{2}}
$$

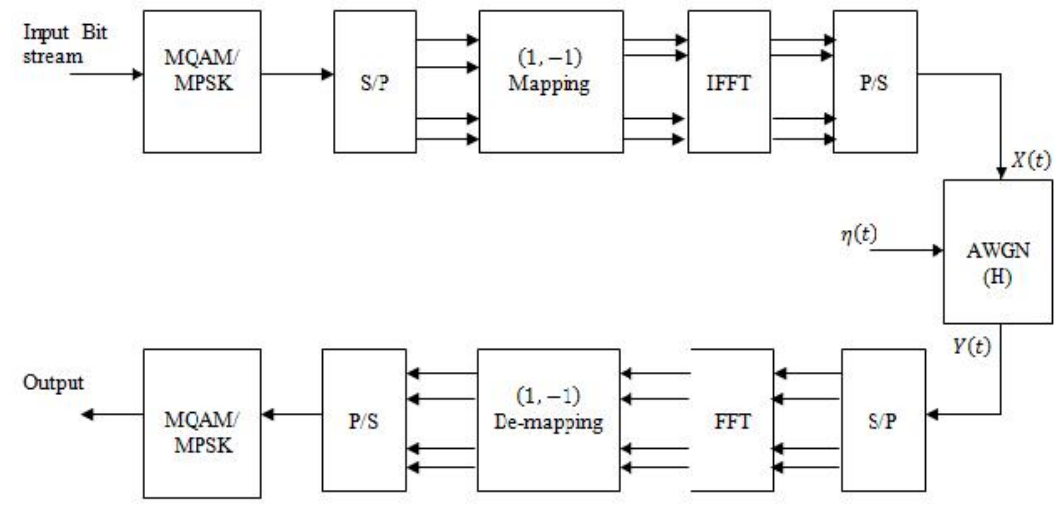

Figure 1. SSR ICI self cancellation scheme

\subsection{Additive White Gaussian Channel}

It adds white gaussian noise to a complex/real input signal. If the input signal is real, then it adds real Gaussian noise and will produces a real output signal. It produces the complex output signal by adding the complex gaussian noise when the input signal is complex. Below are the various modes of noise variance that can be generated by the AWGN Channel: Specifying the Variance Directly or Indirectly

a. Signal-to-Noise ratio $\left(E_{b} / N_{0}\right)$, where the AWGN calculates the variances from these quantities:

- The ratio of energy per bit to noise PSD, $E_{b} / N_{0}$,

- $\quad$ Number of bits per symbol $N_{S}$

- Input signal power

- Symbol period

b. Signal to noise ratio (Es/No), where the AWGN calculates the variances from these quantities:

- Es/No, the ratio between energy of signal to PSD noise

- Input signal power

- Symbol period

c. Signal to noise ratio (SNR), where the AWGN calculates the variances from these quantities

- SNR

- Input signal power

Changing the symbol period in the AWGN Channel will affects the noise variance added per sample, which can also causes a change in the final error rate. 


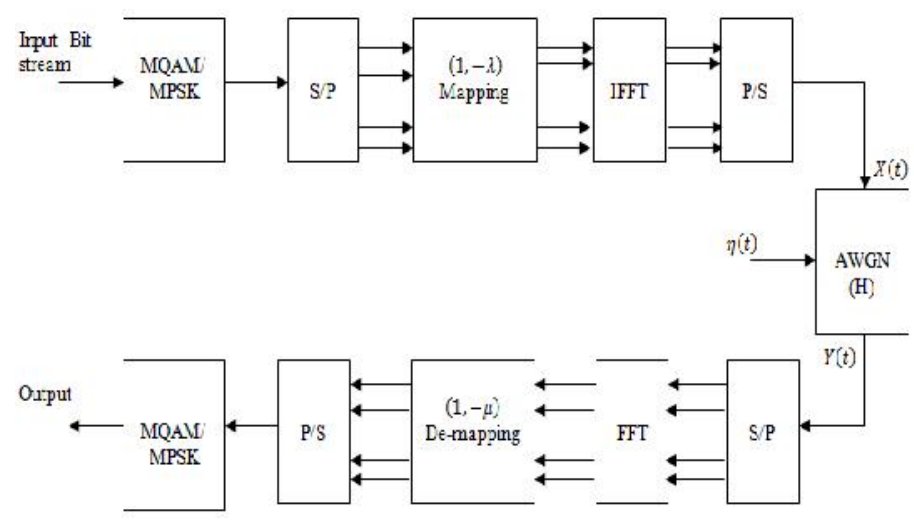

Figure 2. Proposed Block diagram of ICI Self cancellation with AWGN Channel

\section{PROPOSED METHOD}

In the proposed scheme at the transmitter a data allocation $(1,-\lambda)$ is utilized at $k^{\text {th }}$ and $N-1-k^{\text {th }}$ subcarriers .i.e.

$$
X(N-1)=-\lambda X(0), X(N-2)=-\lambda X(1), \ldots X(N-1-k)=-\lambda X(k)
$$

Hence, the received data signal at the $k^{\text {th }}$ subcarrier is

$$
Y^{\prime}(k)=\sum_{i=0}^{\frac{N}{2}-1} X(l) S((l-k)-\lambda S(N-1-l-k))+W(k)
$$

After Combining the received data at $k^{\text {th }}$ and $N-1-k^{\text {th }}$ subcarriers with weight 1 and $-\xi$, we have

$$
\begin{aligned}
& Y^{\prime \prime}(k)=Y^{\prime}(k)-\xi Y^{\prime}(N-1-k) \\
& Y^{\prime \prime}(k)=\sum_{l=0}^{\frac{N}{2}-1} X(l)[S(l-k)-\lambda S(N-1-l-k)-\xi S(l+k+1-N)+\xi \lambda S(k-l)+W(k) \\
& k=0,1,2, \ldots, \frac{N}{2}-1
\end{aligned}
$$

Thus, CIR of proposed optimal SSR ICI self cancellation scheme is given by

$$
C I R_{C}=\frac{|-\xi S(2 k+1-N)+(1+\lambda \xi) S(0)-\lambda S(N-1-2 k)|^{2}}{\sum_{l=0, l \neq k}^{\frac{N}{2}-1}|-\xi S(l-N+k+1)-S(l-k)-\lambda S(N-1-l-k)+\xi \lambda S(l-k)|^{2}}
$$

The optimum values of $\lambda$ and $\xi$ are calculated for $\varepsilon \epsilon[0.03,0.25]$ at a very small interval of $\Delta \varepsilon$ which results in maximum CIR for the given $\varepsilon$. Thus for every $\varepsilon$, we have a unique optimal value of and $\lambda$ and $\xi$ these are denoted by $\left(\lambda_{0}, \mu_{0}\right)$. The optimum values $\left(\lambda_{0}, \mu_{0}\right)$ are to be used for data allocation and combining the data at $k^{\text {th }}$ and $N-1-k^{\text {th }}$ subcarriers to maximize the CIR of the OFDM system. Where, $\operatorname{CIR} p\left(\varepsilon_{1}, \lambda_{01}, \mu_{01}\right)$ corresponds to maximum value of CIR for $\varepsilon_{1}$ and so on and

$$
v=\frac{\left(\varepsilon_{H}-\varepsilon_{L}\right)}{\Delta \varepsilon}+1
$$

Where, $\varepsilon_{H}$ and $\varepsilon_{L}$ are the lowest and the highest possible values of the normalized frequency offset. Here, we have considered $\varepsilon_{H}=0.25$ and $\varepsilon_{L}=0.03$. To avoid the problem of continuous $\varepsilon$ estimation, sub-optimal pair $\left(\lambda_{s o}, \mu_{s o}\right)$ amongst all $\left(\lambda_{0}, \mu_{0}\right)$ has been found by using the following criterion as 


$$
\left(\lambda_{s o}, \mu_{s o}\right)=\max _{\lambda_{0}, \mu_{0}}\left[p-\frac{\sum_{j=1}^{p}\left(p-C I R\left(\varepsilon_{j}, \lambda_{0}, \mu_{0}\right)\right)}{v}\right]
$$

In the above expression, $p$ represents the maximum CIR of aparticular row of the matrix given by (14) and the second term represents the mean deviation of the CIR of that row from the peak (p) of that row. Thus irrespective of the value of $\varepsilon,\left(\lambda_{s o}, \mu_{s o}\right)$ can be used for data allocation and combining to geta sub-optimal CIR performance.

\subsection{Rayleigh Distribution}

Rayleigh fading is a rational model, when an environment that consists of many objects can scatter the transmitted signal before the arrival of signal at receiver. The central limit theorem holds that, the channel impulse response can be modelled well as a gaussian process irrespective of individual components distribution when there are enough much scatter [10]. When we apply Central Limit Theorem (CLT) to the large number of paths, then each path can be modelled with time as the variable as circularly symmetric complex Gaussian random variable (GRV), which is known as Rayleigh channel model [11]. When there is no prevalent component to the scatter such model will have the mean of zero and the phase between 0 and $2 \pi$ radians. Therefore the channel response envelope is Rayleigh distributed.

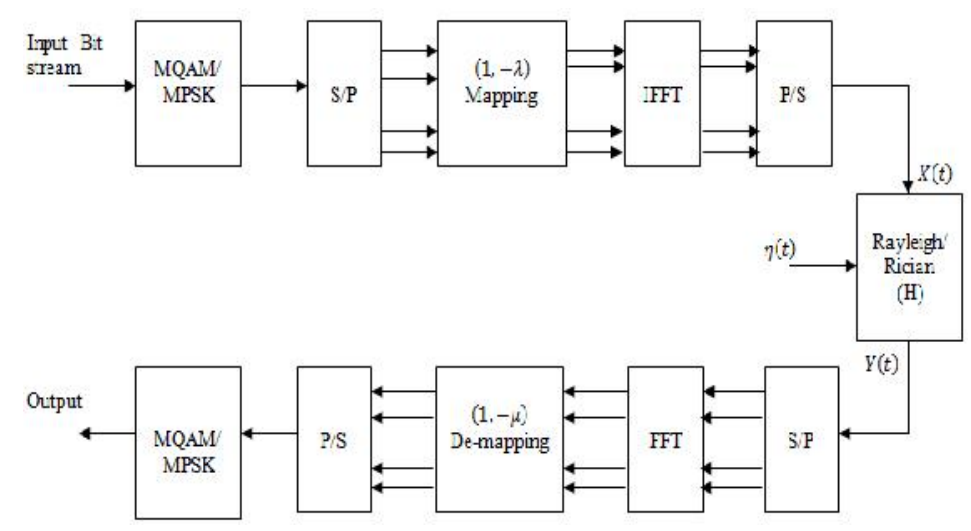

Figure 3. Proposed Block diagram of ICI Self cancellation with Rayleigh and Rician Channel

A circularly symmetric complex GRV is of the form,

$$
Z=X+i J
$$

where the real and imaginary parts are zero mean i.i.d. GRV's.

For circularly symmetric complex random variable,

$$
E[Z]=E\left[e^{j \theta} Z\right]=e^{j \theta}[Z]
$$

A circularly symmetric complex GRV is completely specified by the variance

$$
\sigma^{2}=E\left[Z^{2}\right]
$$

The magnitude $|Z|$, which has the PDF of $\wp(z)$, is called as Rayleigh random variable

$$
\wp(z)=\frac{z}{\sigma^{2}} e^{-\frac{z}{2 \sigma^{2}}}, z>0
$$

\subsection{Rician Channel Distribution}

It occurs when a transmitted signal will deviate from its normal path and cancels itself automatically. It is a non-deterministic model. The transmitted signal can arrive at the receiver end by several different paths, and at least there is change in one path. When the path is much stronger than the others, typically a line of sight (LoS) signal is known as Rician fading (RF). In this, a Rician distribution is used to 
characterize the gain of the amplitude. When there is noLoS path between the transmitter and the receiver of OFDM then the Rayleigh fading [12] can categorize the RF.

RF can be defined by two parameters known as $K$ and $S$. Parameter $K$ is called a Rise factor and it is defined as the ratio between the direct paths power to the other scattered paths power. And the is the total power of both paths, which can acts as a scaling factor for the Rician distribution. The resulting PDF is then given by, $f(x)=\frac{2(K+1) x}{\Omega} \exp \left(-K-\frac{(K+1) x^{2}}{\Omega}\right) I_{0}\left(2 \sqrt{\frac{K(K+1)}{\Omega}} x\right)$

Where the $I_{0}$ is the $0_{t h}$ order modified Bessel function of first kind. If the value of $K$ is zero then the RF envelope will produced down to the Rayleigh faded envelope.

\section{EXPERIMENTAL RESULTS}

In this, we have considered an OFDM system with $\mathrm{N}=64,128$ and 256subcarriers, M-QAM and MPSK modulation schemes tomodulate each of the subcarriers. The simulation model of the existing and proposed schemes with AWGN, Rayleigh and Rician channels is shown in Figure 1, Figure 2 and Figure 3. The computer simulation using MATLAB 2014a are performed to evaluate the Carrier Interference Ratio (CIR) and Bit Error Rate (BER) performance of existing and proposed schemes with respect to the normalized frequency offset and SNR. Figure 4 (a) shows the CIR performance of standard OFDM system, SSR ICI self-cancellation and proposed optimal, sub-optimal approaches with AWGN. It shows that the proposed algorithm under the AWGN channel conditions has highest CIR performance. It has the CIR of $60.23 \mathrm{~dB}$, where the existing and standard OFDM has the CIR values of $44.32 \mathrm{~dB}$ and $33.28 \mathrm{~dB}$. Figure 4 (b) shows BER performance of the standard OFDM system, conventional SSR ICI self cancellation and the proposed approach. As seen from Figure 4 (a) the CIR performance of the proposed optimal approach is about $60.23 \mathrm{~dB}$ far better than the sub-optimal and conventional schemes. The CIR performance of proposed scheme is slightly worse than conventional SSR ICI self cancellation scheme for $\varepsilon \epsilon[0.03,0.25]$. The BER performance of the proposed scheme is very much improved in comparison to standard OFDM system and very close toconventional SSR ICI self cancellation scheme in [3].

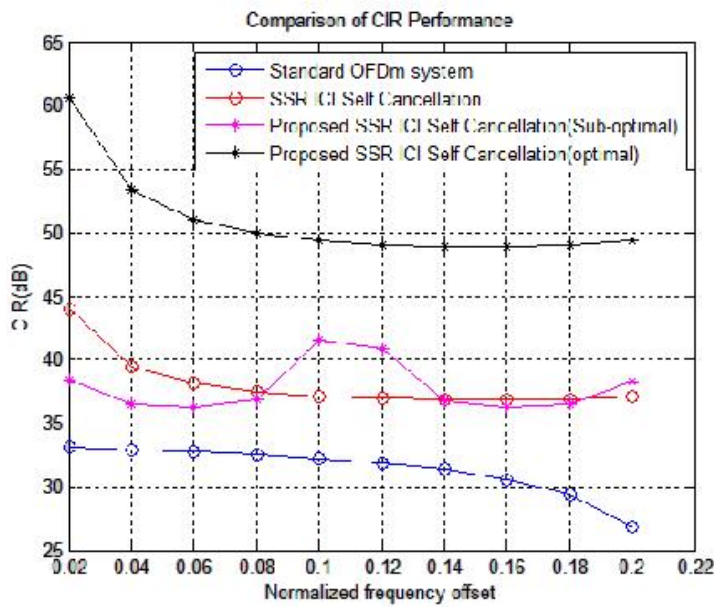

(a)

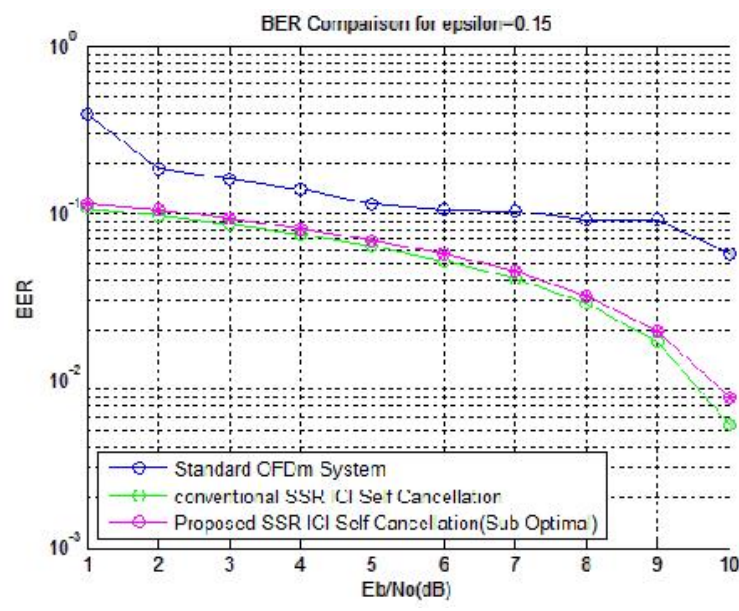

(b)

Figure 4. (a) CIR performance and (b) BER performance Comparison

Figure 5 (a) and (b) shows that the transmitted data and modulated data with 128 subcarriers and 128-PSK. The performance of the proposed scheme with higher modulation levels has shown in figure 6 and figure 7. We tested it with 128-QAM and $\mathrm{N}=128$ subcarriers and we got the CIR of $63.9932 \mathrm{~dB}$, which is an improved performance than the figure 4 results. 


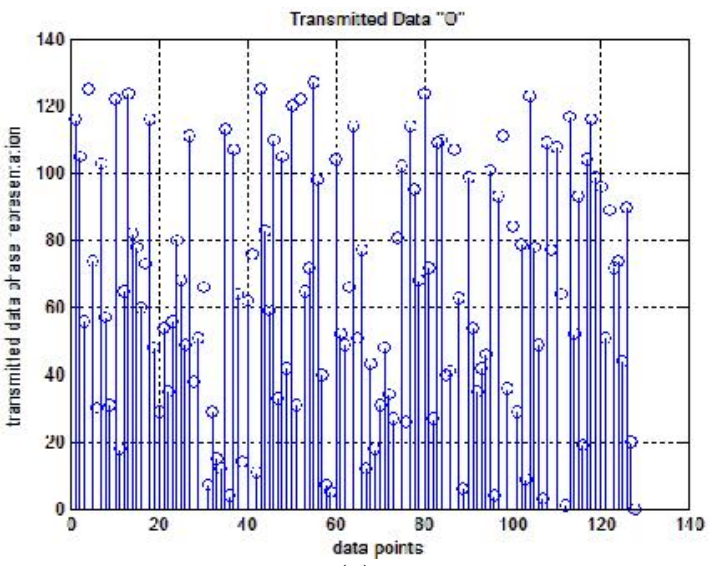

(a)

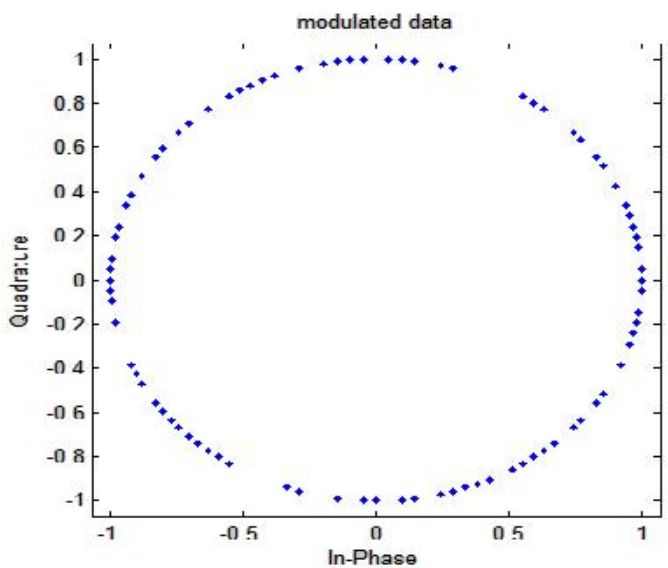

(b)

Figure 5. (a) Data of Transmitter (b) modulated data

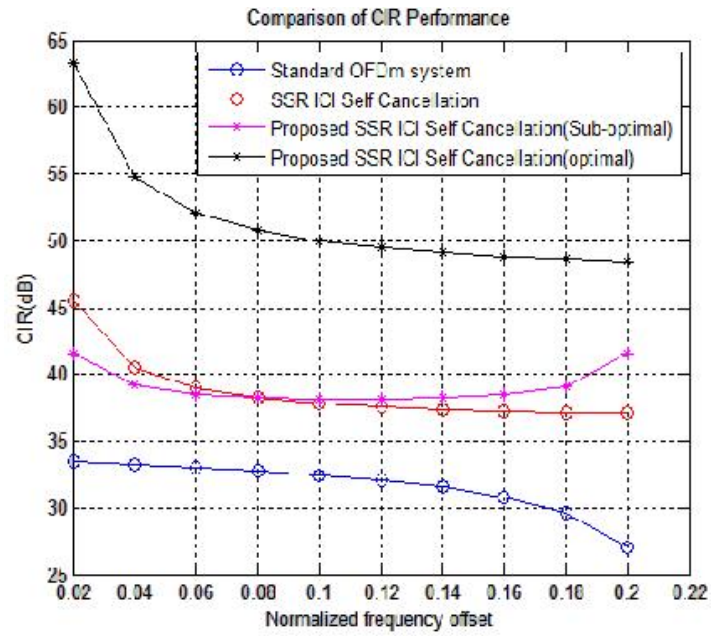

Figure 6. CIR Performance with $\mathrm{N}=128$ and 128QAM

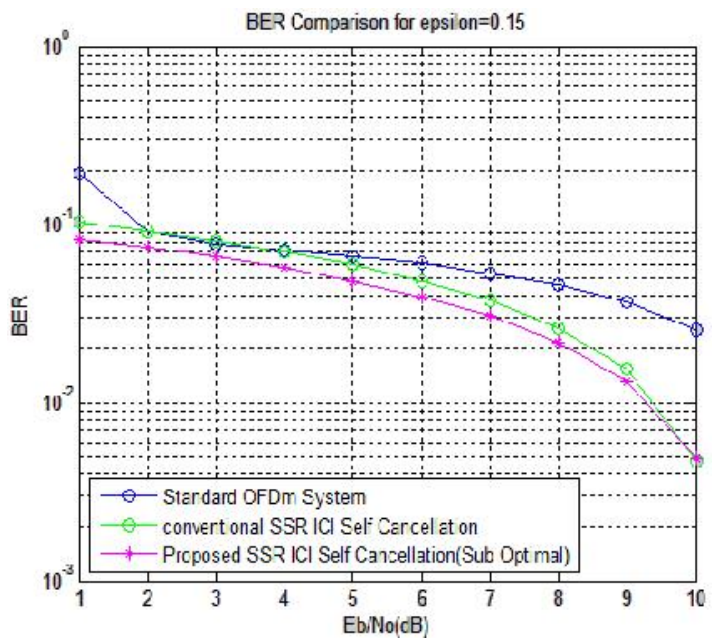

Figure 7. BER performance with 128-QAM and $\mathrm{N}=128$

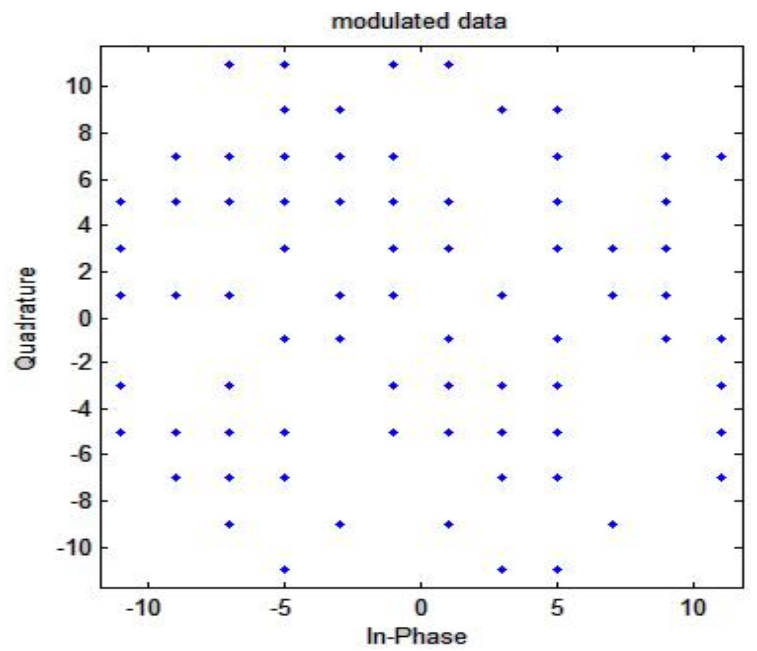

Figure 8. Modulated data with 128-QAM 
The modulated output of 128-QAM in presence of AWGN is shown in fig8 and it has got the CIR which is shown in figure 6.

Further CIR improvement can be achieved by using Rayleigh distribution instead of AWGN. Figure 9 shows the performance of the proposed scheme in presence of Rayleigh channel distribution with 128-PSK and 256 subcarriers. We can see that the proposed scheme has got maximum CIR of 71.325 with the Rayleigh distribution. Figure 8 shows the transmitted data with 256 subcarriers and modulated data with 128-PSK and the figure 10 shows the comparison between the conventional schemes with AWGN and with Rayleigh. It can be observed that while increasing in the frequency offset still the CIR performance stable with the proposed Rayleigh approach and has maximum CIR of 51dB. It's much higher than the other conventional ICI reduction techniques [3-8]. Figure 10 shows the performance of Rician, which has got almost equal results as Rayleigh channel.

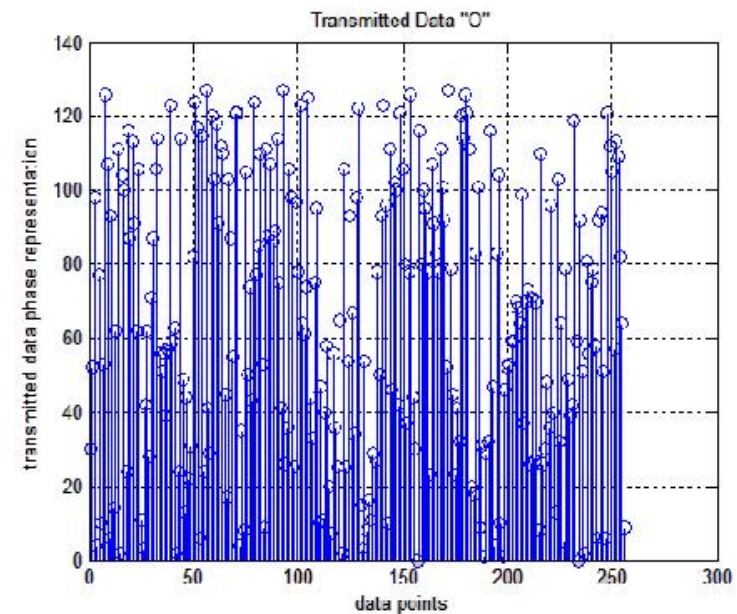

(a)

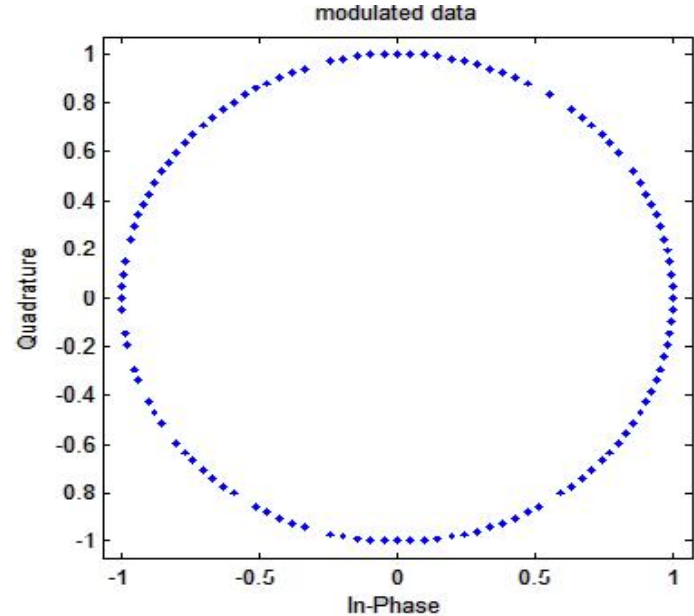

(b)

Figure 9. (a) Transmitted Data of $\mathrm{N}=256$ and (b) modulated data with 128-PSK
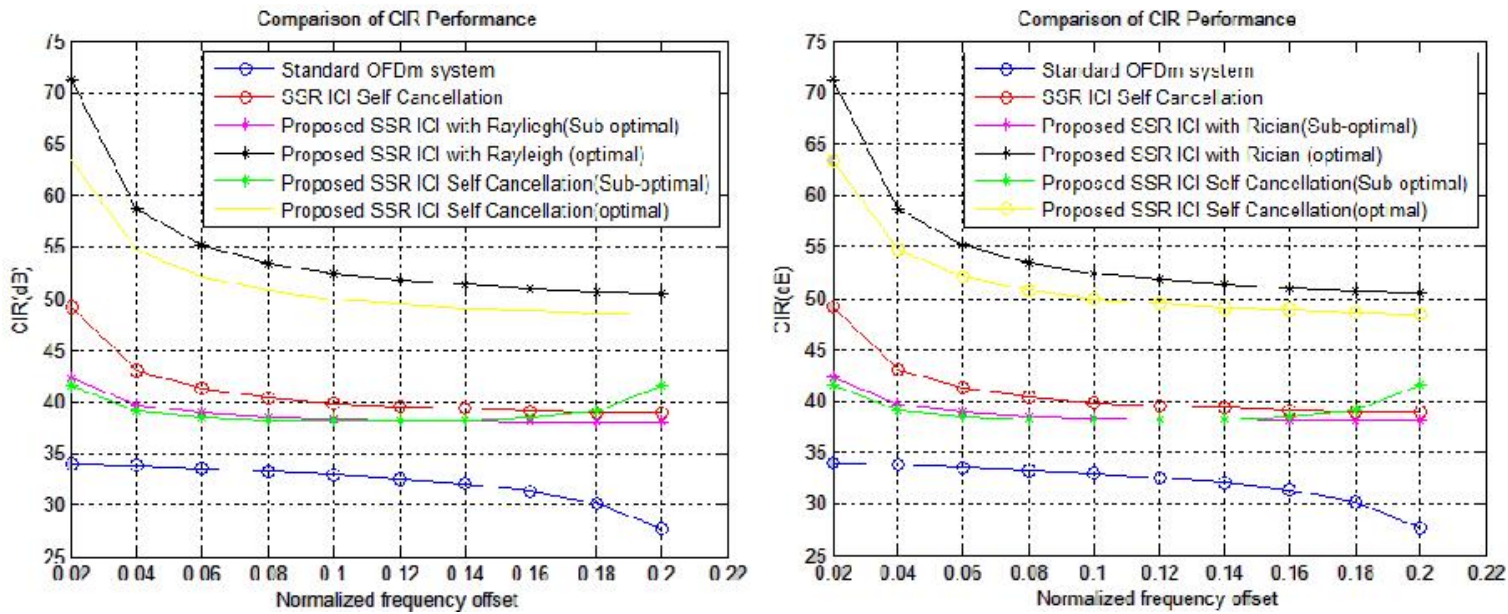

Figure 10. CIR performance with N=256 and 128-PSK under Rayleigh, Ricianchannel models 
Table 1. Simulation parameters

\begin{tabular}{cc}
\hline Parameters & Specifications \\
\hline FFT \& IFFT size & 8 \\
No. of Subcarriers & 64,128 and 256 \\
Cyclic prefix & 1 \\
Channel model & AWGN, Rayleigh and Rician \\
Modulation scheme & QAM, QPSK \\
Constellation points & $4,8,16,32, \ldots$ and 128 \\
OFDM block size & 8 \\
\hline
\end{tabular}

\section{CONCLUSION}

Here in this paper we introduced a new ICI self cancellation algorithm in presence of various channel environments for higher modulation levels with increased subcarriers. And also we had compared the simulation results with the existing algorithms with the proposed scheme under the AWGN, Rayleigh and Rician distributions. After observing the simulation results the Rayleigh and Rician has performed well with $\mathrm{N}=256$ and 128-PSK as well as 128-QAM. We achieved the maximum CIR of $71.325 \mathrm{~dB}$. The proposed scheme well improved the performance of CIR and also decreases the bit error rate with increasing signal to noise ratio values.

\section{REFERENCES}

[1] Y. Wu and W.Y. Zou, "Orthogonal frequency division multiplexing: A multicarrier modualtion scheme", IEEE Transaction on Consumer Electronics, vol. 41, no. 3,pp 392-399, Aug. 1995.

[2] P.H. Moose, "A Technique for orthogonal frequency division multiplexing frequency offset correction, "'IEEE Transactions on Communications, vol. 42, pp 2908-2914, Oct. 1994.

[3] Y. Zhao and S.G. Haggman, "Intercarrier Interference Self-Cancellation Scheme for OFDM Mobile Communication Systems", IEEE Transactions on Communications, vol. 49, no. 7, pp. 1185-1191, Jul. 2001.

[4] H.G. Yeh, Y.K. Chang, and B. Hassibi, "A scheme for cancelling inter carrier interference using conjugate transmission in multicarrier communication systems", IEEE Trans. Wireless Communication., vol. 6, no. 1, pp. 3-7, Jan. 2007.

[5] Chin-Liang Wang and Yu-Chih Huang, "Inter carrier Interference Cancellation Using General Phase Rotated Conjugate Transmission for OFDM Systems", IEEE Transactions on Communications, vol. 58, no. 3, pp. 812-819, March 2010

[6] K. Sathanantham, R.M.A.P. Rajatheva and Slimane Ben Slimane "Analysis of OFDM in the Presence of Frequency Offset and a Method to Reduce Performance Degradation", Global Telecommunications Conference, IEEE, vol. 1,pp. 72-76, 2000

[7] Tureli U., Kivanc D., Hui Liu, "Experimental and Analytical Studies on a High-Resolution OFDM Carrier Frequency Offset Estimator", IEEE Tansactions on Vehicular Technology, Vol. 50, No. 2, pp: 629-643, 2001.

[8] Yu Fu and Chi Chung Ko, "A New ICI Self -Cancellation Scheme for OFDM Systems based on a Generalized Signal Mapper", $5^{\text {th }}$ International Symposium on Wireless Personal Multimedia Communications, vol. 3, pp. 995 999, Oct. 2002.

[9] J.H. Mathews, K.K. Fink, Numerical Methods using Matlab, $4^{\text {th }}$ edition, Prentice-Hall Inc., 2004

[10] Robertson, P.; Kaiser, S. "The effects of Doppler spreads in OFDM (A) mobile radio systems", Vehicular Technology Conference, 1999. VTC 1999 - Fall. IEEE VTS

[11] Bernard Sklar (July 1997). "Rayleigh Fading Channels in Mobile Digital Communication Systems Part I: Characterization". IEEE Communications Magazine 35 (7): 90-100.

[12] Abdi, A. and Tepedelenlioglu, C. and Kaveh, M. and Giannakis, G., "On the estimation of the K parameter for the Rice fading distribution", IEEE Communications Letters, March 2001, p. 92 -94.

[13] S. Popa, N. Drahgiciu, R. Reiz, -Fading Types in Wireless Communications Systems, J. Elect. and Electron. Eng., vol. 1, pp. 232-237, 2008. 


\section{BIOGRAPHIES OF AUTHORS}

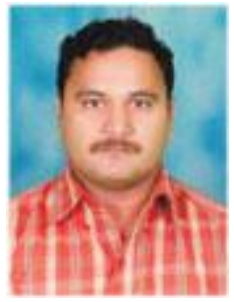

A.H. Sharief obtained his B.E from Osmania University in 1996, M.Tech from JNTU Kakinada in 2010 and is currently pursuing his PhD from Acharya Nagarjuna University.

He is working as Associate Professor in Gandhiji Institute of Science and Technology. His areas of interest include Wireless Communication, Mobile Communications, OFDM.

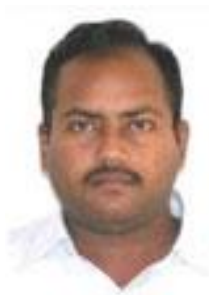

Dr. M. Satya Sai Ram obtained his Ph.D. from JNTU Hyderabad, India. Currently he is working as Professor and Head of the Department in Chalapathi Institute of Engineering and Technology, Guntur, India. He has a large number of technical papers published in national and international journals and conferences. His specializations include Microwave Engineering and Speech Signal Processing. His areas of interest include Communications, Speech Processing and Image Processing. 